\title{
Identification of Thermal Parameters by Treating the Inverse Problem
}

\author{
Abdelaaziz Ghafiri \\ Laboratory of Electronics, \\ Signal Processing and \\ Modelling Physics \\ Department of Physics \\ Ibn Zohr University \\ Agadir ,Morocco \\ El hanafi Arjdal \\ Laboratory of Electronics, \\ Signal Processing and \\ Modelling Physics \\ Department of Physics \\ Ibn Zohr University \\ Agadir, Morocco

\section{Kossi atchonouglo \\ Department of Physics \\ University of Lomé \\ Lome ,Togo}

\author{
Jamal Chaoufi \\ Laboratory of Electronics, \\ Signal Processing and \\ Modelling Physics \\ Department of Physics \\ Ibn Zohr University \\ Agadir ,Morocco
}

\author{
Jean Christophe Dupre \\ Institut $P^{\prime}$ \\ University of Poitiers \\ Poitiers, France
}

\author{
Claude Vallee \\ Institut $P^{\prime}$ \\ University of Poitiers \\ Poitiers, France
}

\author{
Hassan Fatmaoui \\ Laboratory of Electronics, \\ Signal Processing and \\ Modelling Physics \\ Department of Physics \\ Ibn Zohr University \\ Agadir ,Morocco
}

\author{
Arnaud Germaneau \\ Institut $P^{\prime}$ \\ University of Poitiers \\ Poitiers, France
}

\begin{abstract}
The aim of this work is to identify the tensor of thermal conductivity and volumetric heat of an anisotropic material with conductivity tensor diagonal, the heat conduction follows the linear Fourier law. The temperature field in the plate is obtained by solving the analytical heat equation. The solution of the direct problem is simulated by applying the Finite Element Method 2D. The inverse problem is solved by returning the intermediate step of the MEF. An optimization method based on conjugate gradient algorithm has enabled us to develop an identification of thermophysical parameters procedure.
\end{abstract}

\section{Keywords}

Identification; Thermophysical parameters; Inverse problem; Finite Element Method; Infrared Camera; Projected Conjugate Gradient Method

\section{INTRODUCTION}

Improving the characterization of anisotropic materials is currently a major challenge for designing new structures or infrastructures. The thermophysical parameters identification allows to introduce materials whose characteristics meet specific needs or optimize existing systems.

Since the 1980s, several studies have given considerable impetus to inverse methods in thermal: Hensel applies reverse engineering theories [1] Kurpisz determines the heat flux [2], Ballis measuring the thermal diffusivity of cell carbon foam [3]. Some authors have studied the sensitivity of the parameters $\lambda$ and $\rho c$ [3] [4] [5]. In the literature, few studies intended to. the simultaneous identification of the specific heat and conductivity Atchongolo presented a method for identifying thermal parameters for isotropic materials [6]

The approach of this study is based on the exploitation of temperature fields obtained by the analytical resolution of the heat equation. The Inverse Problem is solved by crossing back the equations obtained by the Finite Element Method for solving the Direct $\mathrm{P}$ identify the thermal parameters of an material with thermal conductivity tensor is diagonal $\lambda=\left[\begin{array}{cc}\lambda_{1} & 0 \\ 0 & \lambda_{2}\end{array}\right]$.

To validate our calculation approach we compared the temperature fields determined, analytically and numerically, with the experimental fields temperatures measured by an infrared camera on a plate of polymethylmethacrylate (PMMA), these measures were carried out within the institute P' of the University of Poitiers.

\section{DIRECT PROBLEM}

\subsection{Position of the problem}

In order to identify the thermal characteristics of a material, we performed a procedure both experimental and numerical. We consider a rectangular thin solid plate of length $L$, width $l$ and thickness $e$. The plate is homogeneous, its thickness is small and the length is very close to the width. We will assume that the temperature distribution is two-dimensional.

Let us consider that the plate occupies the interval $[0, L]$ from the $O x$ axis, and $[0, l]$, by the $0 y$ axis. The temperature distribution within the plate is a function $T(x, y, t)$ of 
coordinates $(x, y)$ and time $t$. At time $t=0$, the temperature distribution is known, we will denote it $T_{0}(x, y)$. A constants heat flux $q_{1}$ is imposed on the side bounded by $x=L$ (denoted $\Gamma_{3}$ ) and $q_{2}$ is imposed on the side bounded by $y=l$ (denoted $\left.\Gamma_{4}\right)$. The other sides (denoted $\Gamma_{1}$ and $\Gamma_{2}$ ) are well protected against any convective, radiative or conductive currents. Figure 1 illustrates the domain $\Omega=[0, L] \times[0, l]$ occupied by the plate and the boundary conditions.

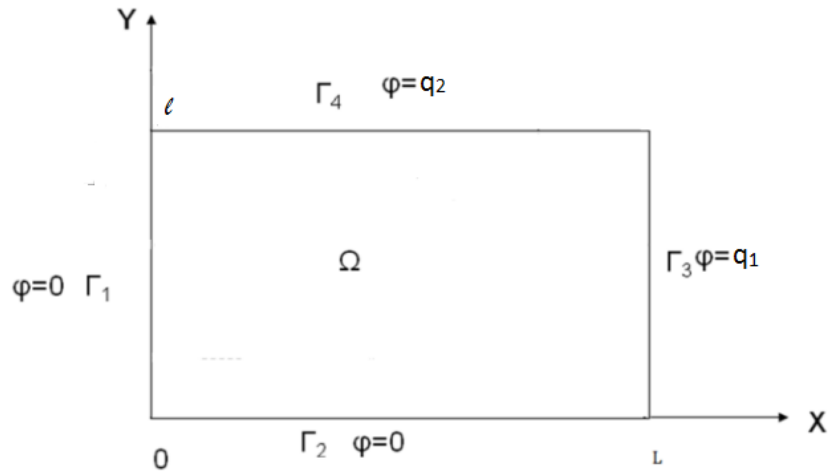

Fig 1: Rectangular plate and boundary conditions

Let $\rho c$ be the heat capacity per unit volume ( $\rho$ being the specific mass and $c$ the heat capacity per unit mass). Let $\lambda=\left[\begin{array}{cc}\lambda_{11} & 0 \\ 0 & \lambda_{22}\end{array}\right]$ be the thermal conductivity tensor. According to Fourier's prescriptions, this symmetrical tensor is assumed to be positive definite. The temperature distribution $T(x, y, t)$ in the plate is governed by the diffusion equation [7]:

$\rho c \frac{\partial T}{\partial t}=\lambda_{1} \frac{\partial^{2} T}{\partial x^{2}}+\lambda_{2} \frac{\partial^{2} T}{\partial y^{2}} \quad$ in $\quad \Omega=[0, L] \times[0, l]$

The above boundary conditions read:

$\lambda_{1} \frac{\partial T}{\partial x}(0, y, t)=0 \quad \lambda_{1} \frac{\partial T}{\partial x}(L, y, t)=q_{1}$

$\lambda_{2} \frac{\partial T}{\partial y}(x, 0, t)=0 \quad \lambda_{2} \frac{\partial T}{\partial y}(x, l, t)=q_{2}$

The initial condition reads:

$$
T(x, y, 0)=T_{0}(x, y)=0 .
$$

\subsection{Analytical resolution of the direct problem}

A particular solution of the heat equation and the boundary conditions is

$$
\theta(x, y, t)=\frac{1}{\rho C}\left(\frac{q_{1}}{L}+\frac{q_{2}}{l}\right) t+\frac{q_{1}}{2 \lambda_{1} L}\left(x^{2}-\frac{L^{2}}{3}\right)+\frac{q_{2}}{2 \lambda_{2} l}\left(y^{2}-\frac{l^{2}}{3}\right)
$$

but this solution does not satisfy the initial condition. we will use these functions $\frac{q_{1}}{2 \lambda_{1} L}\left(x^{2}-\frac{L^{2}}{3}\right)$ and $\frac{q_{1}}{2 \lambda_{1} l}\left(\mathrm{y}^{2}-\frac{l^{2}}{3}\right)$ instead functions $\frac{q_{1}}{2 \lambda_{1} L} x^{2}$ and $\frac{q_{1}}{2 \lambda_{1} l} y^{2}$ because they are null mean (in $\mathrm{x}$, respectively in $\mathrm{y}$ ). the function $v(x, y, t)=T(x, y, t)-\theta(x, y, t)$ is the solution of the heat equation with all the homogeneous boundary conditions and the initial condition

$$
v(x, y, 0)=-\frac{q_{1}}{2 \lambda_{1} L}\left(x^{2}-\frac{L^{2}}{3}\right)-\frac{q_{2}}{2 \lambda_{2} l}\left(y^{2}-\frac{l^{2}}{3}\right)
$$

The method of separation of variables leads to search $v(x, y, t)$ in the form of a double Fourier series

$$
\begin{gathered}
v(x, y, t)=\sum_{m=0}^{\infty} \sum_{n=0}^{\infty} A_{m n} \exp \left[-\frac{\pi^{2} t}{\rho C}\left(\frac{\lambda_{1} m^{2}}{L^{2}}+\frac{\lambda_{2} n^{2}}{l^{2}}\right)\right] \\
\cos \frac{m \pi x}{L} \cos \frac{n \pi y}{l}
\end{gathered}
$$

At time 0 , the exponential is worth 1 , the coefficients $A_{m n}$ are determined by the initial condition.

$$
\begin{aligned}
& \sum_{m=0}^{\infty} \sum_{n=0}^{\infty} A_{m n} \cos \frac{m \pi x}{L} \cos \frac{n \pi y}{l} \\
& =-\frac{q_{1}}{2 \lambda_{1} L}\left(x^{2}-\frac{L^{2}}{3}\right)-\frac{q_{2}}{2 \lambda_{2} l}\left(y^{2}-\frac{l^{2}}{3}\right)
\end{aligned}
$$

With our choice of functions with zero mean in $\mathrm{x}$ and in $\mathrm{y}$, the coefficient $A_{00}$ is null and only the coefficients $A_{0 m}$ and $A_{n 0}$ $(m \geq 1, n \geq 1)$ are not null.

$$
\begin{aligned}
v(x, y, t)= & \sum_{m=1}^{\infty} A_{m 0} \exp \left(-t \frac{\lambda_{1} \pi^{2} m^{2}}{\rho C L^{2}}\right) \cos \frac{m \pi x}{L} \\
& +\sum_{n=1}^{\infty} A_{0 n} \exp \left(-t \frac{\lambda_{2} \pi^{2} n^{2}}{\rho C l^{2}}\right) \cos \frac{n \pi y}{l}
\end{aligned}
$$

We integrate twice by parts

$$
\begin{gathered}
\frac{m \pi}{L} \int_{0}^{L} x^{2} \cos \frac{m \pi x}{L} d x=2 \frac{L^{2}}{m \pi}(-1)^{m} \\
\int_{0}^{L} \cos ^{2}\left(\frac{m \pi x}{L}\right) d x=\frac{L}{2}
\end{gathered}
$$

Such as

thus $\frac{L}{2} A_{m 0}=-\frac{q_{1}}{\lambda_{1}} \frac{L^{2}}{m^{2} \pi^{2}}(-1)^{m}$ and $\frac{l}{2} A_{0 n}=-\frac{q_{2}}{\lambda_{2}} \frac{l^{2}}{m^{2} \pi^{2}}(-1)^{n}$

Finally the temperature in the plate is

$$
\begin{aligned}
& T(x, y, t)=\frac{1}{\rho C}\left(\frac{q_{1}}{L}+\frac{q_{2}}{l}\right) t+\frac{q_{1} L}{2 \lambda_{1}}\left(\frac{x^{2}}{L^{2}}-\frac{1}{3}\right)+\frac{q_{2} l}{2 \lambda_{2}}\left(\frac{y^{2}}{l^{2}}-\frac{1}{3}\right) \\
& -\frac{2 q_{1} L}{\lambda_{1}} \sum_{m=1}^{\infty} \frac{(-1)^{m}}{m^{2} \pi^{2}} \exp \left(-t \frac{\lambda_{1} \pi^{2} m^{2}}{\rho C L^{2}}\right) \cos \frac{m \pi x}{L} \\
& -\frac{2 q_{2} l}{\lambda_{2}} \sum_{n=1}^{\infty} \frac{(-1)^{n}}{n^{2} \pi^{2}} \exp \left(-t \frac{\lambda_{2} \pi^{2} n^{2}}{\rho C l^{2}}\right) \cos \frac{n \pi y}{l}
\end{aligned}
$$

In the next part we will give an alternative method for solving the heat equation using the finite element method (FEM).

\subsection{Resolution of the Direct problem by Finite Element Method(FEM)}

The above Partial Differential Equation (1) admits a variational formulation. The latter consists in multiplying the heat conduction equation by a regular test function $T^{*}$, and to integrate it over the whole domain $\Omega$. This weak formulation leads to solve the equivalent problem [5]: Among 
all temperature fields satisfying the initial condition $T(x, y, 0)=T_{0}(x, y)$, find the one for which

$\int_{\Omega} \rho c T^{*} \frac{\partial T}{\partial t} d x d y+\int_{\Gamma_{1}} T^{*} \varphi_{s} d y+\int_{\Omega}\left(\operatorname{grad} T^{*}\right) \cdot(\lambda \operatorname{grad} T) d x d y=0$ and this for any test function $T *$.

An approximate solution of the diffusion problem presented in paragraph 2.3 can be obtained by choosing a finite number of appropriate test functions. We present next some details about this approach for obtaining the temperature field in the framework of Finite Element Method.

The Finite Element Method [8] is applied for the space discretization of the partial differential equations. We proceed to the discretization of the domain $\Omega$ into $\mathrm{Nt}$ three nodes triangular elements $\Omega^{e}$. The total number of nodes is denoted $\mathrm{N}$ [9]. Let us denote $\left[A^{e}\right]$ the $n^{e} \times n_{t}$ positioning matrix defined by the following rule: a component $\left[A^{e}\right]_{i j}$ is equal to 1 if the node numbered globally $j$ on the structure coincides with the node numbered locally $i$ on the element e, it is equal to 0 otherwise. The matrix $\left[B^{e}\right]$ is the gradient matrix of element (e).

Therefore, this boundary value problem reduces to the ordinary differential equation (ODE).

$$
[C]\left[\frac{d T}{d t}\right]+\sum_{e}\left[B^{e} A^{e}\right] \lambda\left[B^{e} A^{e}\right]^{t}[T]=[F]
$$

We can summarize this ODE issued from a weak formulation associated to a spatial discretization as [6]:

$$
[C]\left\{\frac{d T}{d t}\right\}+[K]\{T\}=\{F\}
$$

where

$$
\begin{aligned}
& -\quad\{\mathrm{T}\} \text { is the nodal temperature vector, } \\
& -\quad[\mathrm{C}] \text { is the overall capacity matrix, } \\
& -\quad[\mathrm{K}] \text { is the overall conductivity matrix, } \\
& -\quad[\mathrm{F}] \text { is the overall nodal flux vector. }
\end{aligned}
$$

Solving our problem of transient conduction by FEM leads into solving the first order ODE for which the initial condition is: $\left[T_{0}\right]^{t}=\left[\begin{array}{llll}T_{1}(0) & T_{2}(0) & \ldots & T_{N}(0)\end{array}\right]$. The temperature field in the material will be approximated at the nodes.

\subsection{Results of the direct problem}

In this part, and to validate our approach to calculating we compared the analytical and numerical results with experimental temperatures for PMMA plate with the following characteristics [10]

$$
\begin{aligned}
& L=0,148 m \text { and } l=0.041 \\
& - \text { Discretization step: } \Delta x=L / 148 \text { and } \\
& \Delta y=l / 41 \\
& - \text { Thermal } \\
& \bar{\lambda}=\left[\begin{array}{cc}
0.17 & 0 \\
0 & 0.17
\end{array}\right]\left(\mathrm{W} / \mathrm{m} /{ }^{\circ} \mathrm{C}\right)
\end{aligned}
$$$$
\text { - Dimensions of the plate: }
$$

tensor:

$$
\begin{aligned}
& \text { - Specific heat } \rho c=1,666.10^{6} \mathrm{~J} / \mathrm{m}^{3} /{ }^{\circ} \mathrm{C} \\
& \text { - Flows } q_{1}=256 \mathrm{~W} / \mathrm{m} \text { and } q_{2}=0 \\
& \text { - Time step: } \quad \Delta t=1 \mathrm{~s}
\end{aligned}
$$

Figure 2 below illustates the evolution of experimental and simulated temperatures of the plate, at a node with respect to time.

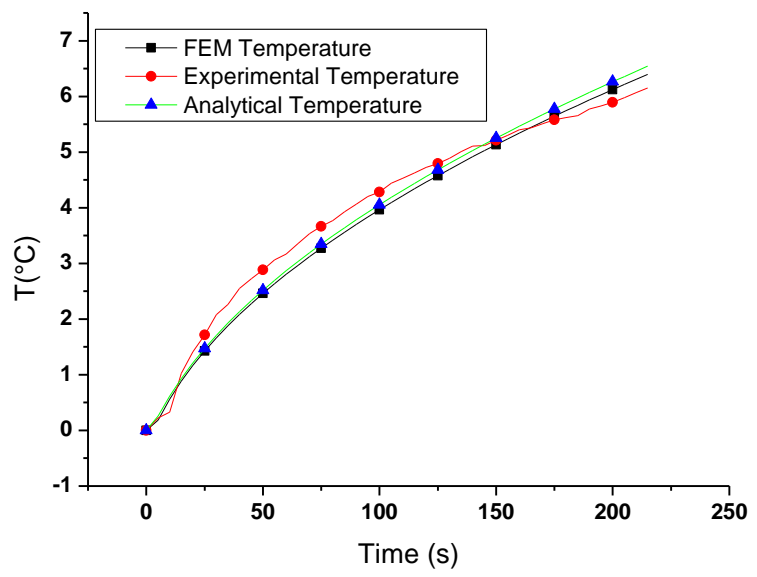

Fig 2: Evolution of temperature at point of coordinates $(0.147,0.02)$

Figure 3 below illustates the evolution of experimental and simulated temperatures of the plate, in a horizontal line at time $\mathrm{t}=100 \mathrm{~s}$ and $\mathrm{y}=0.02 \mathrm{~m}$.

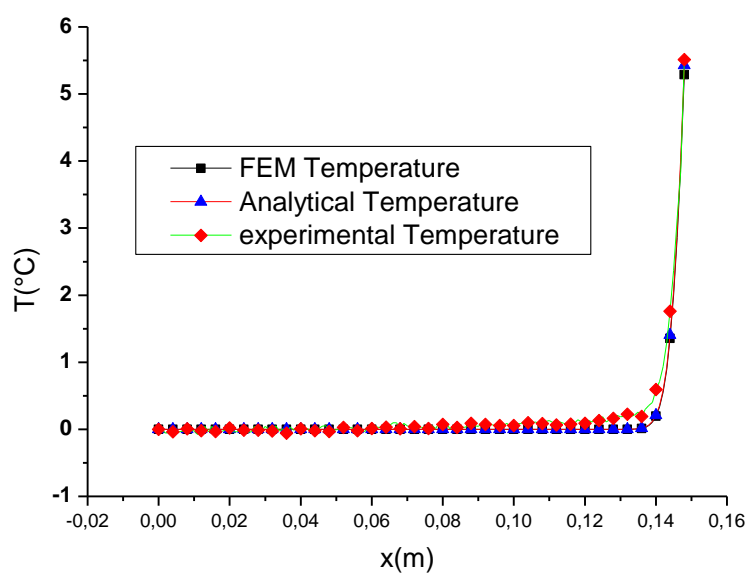

Fig 1: Changes of the temperature in function of the abscissa $\times(t=100 \mathrm{~s}, \mathrm{y}=0.02 \mathrm{~m})$

Note that the simulated analytical temperature evolution curves fit the experimental ones, This comparison validates the developed direct approach.

Next, in the inverse problem and to put in the experimental conditions, a noise with standard deviation $\sigma=0.02^{\circ} \mathrm{C}$ is imposed on the temperature according to the precision current infrared cameras

\section{INVERSE PROBLEM}

\subsection{Position of the problem}

In the inverse problem we enjoy the results obtained from the analytical solution of the direct problem to estimate the 
thermo physical parameters of a given material. Once the temperature function $\mathrm{T}(\mathrm{x}, \mathrm{y}, \mathrm{t})$ is recorded in a series of points on the surface of the sheet, repeatedly, the least squares method is applied to estimate thermodynamic parameters. The method of least squares leads to constrained optimization process: Reduce the cost function

$$
J(\rho c, \lambda)=\frac{1}{2} \int_{0}^{t_{f}}\left\|[C]\left\{\frac{d T}{d t}\right\}+\sum_{e}\left[B^{e} A^{e}\right] \lambda\left[B^{e} A^{e}\right]^{t}\{T\}-\{F\}\right\|^{2} d t
$$

Under the positive constraint for $\rho c$, and the diagonal constraint for $\lambda$.

To minimize this function by a steepest descent method, we need to express its gradient with respect to the conductivity tensor $\lambda$ :

$$
R=\frac{1}{2} \int_{0}^{t_{f}} \sum_{e}\left[B^{e} A^{e}\right]\left[U\{T\}^{t}+\{T\}(U)^{t}\right]\left[B^{e} A^{e}\right]^{t} d t
$$

with

$$
U=[C]\left\{\frac{d T}{d t}\right\}+[K]\{T\}-\{F\}
$$

We will need also its derivative with respect to the specific

$$
\text { heat: } r=\frac{\partial J}{\partial(\rho c)}
$$

\subsection{Identification algorithm}

As steepest descent method to minimize the function $J(\rho c, \lambda)$, we implement the projected conjugate gradient method which consists in constructing iteratively a sequence converging to the minimum. .

The algorithm of this method can be summarized as follows

1. Initialize $\lambda$ by $\lambda_{0}$ and $\rho c$ by $(\rho c)_{0}$,

Deduce the initial values $r_{0}$ and $R_{0}$ of $r$ and $R$,

Initialize a sequence of scalars $d_{i}$ by $d_{0}=-r_{0}$ and a sequence of directions $D_{i}$ by $D_{0}=-R_{0}$,

2. At iteration $i$

calculate $\mu_{i}$ and $v_{i}$ which minimize

$$
\begin{aligned}
& J\left((\rho c)_{i}+\mu d_{i}, \lambda_{i}+v D_{i}\right) \text { with respect to } \mu \text { and } v \\
& (\rho c)_{i+1}=(\rho c)_{i}+\mu_{i} d_{i} \\
& \lambda_{i+1}=\lambda_{i}+v_{i} D_{i} \\
& \text { 3. if }\left\|r_{i+1}\right\|<\varepsilon \text { and }\left\|R_{i+1}\right\|<\varepsilon \text { stop, otherwise } \\
& d_{i+1}=-r_{i+1} \\
& \beta_{i}=\frac{\left(R_{i+1}-R_{i}\right)^{t} R_{i}}{R_{i}^{t} R_{i}} \\
& D_{i+1}=-R_{i+1}+\beta_{i} D_{i} \\
& i=i+1 \text { and return to step 2. }
\end{aligned}
$$

In step 3, the test stop is based on a preassigned small scalar $\varepsilon$, the calculus of $\beta_{i}$ follows the prescription of Polak and Ribière [11].

\subsection{Identification results}

The Conjugate Gradient method developed in the last section is applied to the simulated temperature fields obtained by analytical resolution of the direct problem. The material is supposed to be anisotropic with diagonal thermal conductivity tensor. The results from our identification algorithm without noise are shown in the table 1 below.

Table 1. Identified Values from simulated temperature fields without noise

\begin{tabular}{|c|c|c|}
\hline Parameters & $\begin{array}{c}\text { Values used in } \\
\text { the simulation }\end{array}$ & identified Values \\
\hline$\lambda_{\mathbf{1}}\left(\mathbf{W} / \mathbf{m} /{ }^{\circ} \mathbf{C}\right)$ & 0.45 & 0.4309 \\
\hline$\lambda_{\mathbf{2}}\left(\mathbf{W} / \mathbf{m} /{ }^{\circ} \mathbf{C}\right)$ & 0.17 & 0.1687 \\
\hline $\boldsymbol{\rho c}\left(\mathbf{J} / \mathbf{m} 3{ }^{\circ} \mathbf{C}\right.$ & $1.666 .10^{6}$ & $1.6759 .10^{6}$ \\
\hline
\end{tabular}

The table 2 shows the values identified from the noisy temperatures

Table 2. Identified Values from simulated noisy temperature fields

\begin{tabular}{|c|c|cc|}
\hline Parameters & $\begin{array}{c}\text { Values used in } \\
\text { the simulation }\end{array}$ & \multicolumn{2}{|c|}{ identified Values } \\
\hline$\lambda_{\mathbf{1}}\left(\mathbf{W} / \mathbf{m} /{ }^{\circ} \mathbf{C}\right)$ & 0.45 & 0.4309 & \pm 0.0170 \\
\hline$\lambda_{2}\left(\mathbf{W} / \mathbf{m} /{ }^{\circ} \mathbf{C}\right)$ & 0.17 & 0.1638 & \pm 0.0140 \\
\hline $\boldsymbol{\rho c}\left(\mathbf{J} / \mathbf{m} 3 /{ }^{\circ} \mathbf{C}\right)$ & $1.666 .10^{6}$ & $1.6573 .10^{6}$ & $\pm 0.027 .10^{6}$ \\
\hline
\end{tabular}

\section{CONCLUSION}

The finite element method meets the requirements imposed by the sample geometry and the boundary conditions. Its application on a homogeneous anisotropic material enabled us to transform the Fourier's heat conduction equation in a first order ordinary differential equation. Therefore, the resolution of the direct problem needs solely a time integration algorithm. The developed algorithm allows us to simulate the temperature field in the bidimensional case. The accuracy of the simulations ensured the validity of our approach. Moreover, our code proved to be fast handling, as well for varied geometric dimensions,, than for varied boundary and initial conditions.

The identification algorithm is based on the conjugate Gradient method. It allows to characterize the thermal conductivity tensor and the specific heat. of materials. The identification results revealed to be in good agreement with the values used in the simulation of the direct problem.

\section{REFERENCES}

[1] Hensel E., Inverse theory and applications for engineers, Prentice-Hall INC, Englewood Cliffs,N. J., 1978

[2] Kurpisz K., Nowak A. J., Inverse Thermal Problems, Computational Mechanics, 1995.

[3] Baillis D., Laurent M., Raynaud M., Gervais P., « Méthode Flash appliquée aux mousses de carbone », Congrès Français de Thermique, Lyon, 15-17mai, 2000.

[4] Ammous A., Sellami F., Ammous K.,Morel H., Allard B., Chante J.-P., « Developing an equivalent thermal model for discrete semiconductor packages », International Journal of Thermal Sciences, vol. 42, p. 533-539, 2003.

[5] Vozàr L., Srámková T., « Two date reduction methods for evaluation of thermal diffusivity from step-heating 
measurement », Int. J. Heat Mass Transfert, vol. 40, n_ 7, p. 1647-1657, 1997.

[6] Atchonouglo K., Identification des Paramètres Caractéristiques d'un Phénomène Mécanique ou Thermique Régi par une équation différentielle ou aux dérivées Partielles, Thèse de Doctorat, Université de Poitiers, 2007. .

[7] Sacadura J.F., Initiation aux transferts thermiques, Technique et documentation, 1993

[8] Bergheau J.M., Fortunier R., Simulation numérique des transferts thermiques par éléments finis, Hermès, Paris, 2004.
[9] Aouragh B., Chaoufi J., Fatmaoui H., Dupré J.C., Vallée C., Atchonouglo K., Simulation of thermo mechanical behavior of structures by the numerical resolution of direct problem, Applied Mechanics and Materials, vol. 61, 85-93, 2011..

[10] Atchonouglo K., Banna M., Vallée C., ,Dupré J.C, Inverse Transient Heat Conduction Problems and Application to the Estimation of Heat Transfer Coefficients, Heat and Mass Transfer, vol. 45, issue 1, 23-29, 2008.

[11] Polak E., Computational Methods in Optimization: A Unified Approach, R. Bellman, Ed., Mathematics in Science and Engineering, vol. 77, New York, NY: Academic Press, 1971. 